\title{
A Computational Study of Molecular Diffusion and Dynamic Flow through Carbon Nanotubes
}

\author{
Zugang Mao and Susan B. Sinnott* \\ Department of Chemical and Materials Engineering, University of Kentucky, Lexington, Kentucky 40506-0046
}

Received: December 17, 1999; In Final Form: March 6, 2000

\begin{abstract}
Molecular dynamics simulations are used to study the flow of methane, ethane, and ethylene through carbon nanotubes at room temperature. The interatomic forces in the simulations are calculated using a classical, reactive, empirical bond-order hydrocarbon potential coupled to Lennard-Jones potentials. The simulations show that the intermolecular and molecule-nanotube interactions strongly affect both dynamic molecular flow and molecular diffusion. For example, molecules with initial hyperthermal velocities slowed to thermal velocities in nanotubes with diameters less than $36 \AA$ A In addition, molecules moving at thermal velocities are predicted to diffuse from areas of high density to areas of low density through the nanotubes. Normalmode molecular thermal diffusion is predicted for methane for nearly all the nanotube diameters considered. In contrast, ethane and ethylene are predicted to diffuse by normal mode, single-file mode, or at a rate that is transitional between normal-mode and single-file diffusion over the time scales considered in the simulations, depending on the diameter of the nanotube. When the nanotube diameters are between 16 and $22 \AA$, ethane and ethylene are predicted to follow a helical diffusion path that depends on the helical symmetry of the nanotube. The effects of atomic termination at the nanotube opening and pore-pore interactions within a nanotube bundle on the diffusion results are also considered.
\end{abstract}

\section{Introduction}

Many research groups have studied carbon nanotubes since they were first synthesized in $1991 .^{1}$ Because of their nanometerscale size and hollow, cylindrical shape, they have many potential applications as molecular sieves, nano-test tubes, and hydraulic actuators. Nanotubes have therefore been proposed ${ }^{2}$ as promising materials for the production of tailored ultrafiltration membranes (membranes with pores on the order of $1-100 \mathrm{~nm}) .{ }^{3}$ Usually, these membranes are synthesized by only partially sintering a ceramic or by stretching a polymer to create pores with the desired density, size, and shape. ${ }^{3}$ A carbon nanotube membrane might be constructed of chemically opened nanotubes $^{4-6}$ that are arranged in a close-packed structure (bundle).

It has been well established that in porous materials diffusive flow is dominant in nanometer-scale pores. ${ }^{7}$ Diffusion in such restricted structures is therefore of interest and is expected to be important for numerous applications such as shape selective catalysis $^{8}$ and separations. ${ }^{9-13}$ Until recently, ${ }^{14}$ technical challenges have prevented the direct study of molecular diffusive flow in materials with nanometer-scale pores. Therefore, numerous computational molecular dynamics (MD) and/or Monte Carlo (MC) studies have been conducted on molecular diffusion in various ideal nanopores ${ }^{15}$ and zeolites. ${ }^{16-22}$

Carbon nanotubes are similar to zeolites and other molecular sieves in that they contain nanometer-scale pores. However, this does not necessarily mean a priori that atomic and molecular diffusion in nanotubes will be the same as in zeolites. For example, nanotubes have continuous, smooth walls of uniform composition, whereas zeolites generally have varying diameters and are composed of multiple elements. In addition, variations

*Corresponding author. E-mail: sinnott@engr.uky.edu. in the helical arrangement of the carbon atoms along the nanotube axis could influence molecular motion.

Several groups have studied intercalation inside carbon nanotubes. For example, experiments show that numerous compounds and elements with low surface tensions intercalate into opened carbon nanotubes, ${ }^{23,24}$ but only into those nanotubes with diameters large enough to accommodate capillary motion. ${ }^{25}$ In addition, first principles calculations predict that it is energetically favorable for HF molecules to intercalate into small carbon nanotubes. ${ }^{26}$ Several studies ${ }^{27-29}$ have been made of hydrogen adsorption in nanotube bundles, a problem of particular technological importance for applications that rely on stored hydrogen fuel. Also, simulations of the dynamic flow of helium and argon atoms through nanotubes ${ }^{30}$ have predicted that the flow slows rapidly at low temperatures and that the heavier argon slows more quickly than the helium atoms. Finally, recent MC simulations have predicted quantum sieving of hydrogen and neon molecules in carbon nanotubes with diameters less than $7 \AA .{ }^{31}$

To better understand the movement of polyatomic molecular species through carbon nanotubes, we have modeled the dynamic and diffusive flow of methane, ethane, and ethylene molecules through single-walled carbon nanotubes, where the dynamic flow study is the nanometer-scale analogue of macroscopic-scale fluid flow through pipes. Nanotubes of various diameters and helical structures were considered in the simulations, all of which were performed at $300 \mathrm{~K}$.

\section{Computational Details}

The computational approach used was classical MD simulations, where Newton's equations of motion are numerically integrated with a third-order Nordsieck predictor corrector integration scheme to track the motion of the atoms with 


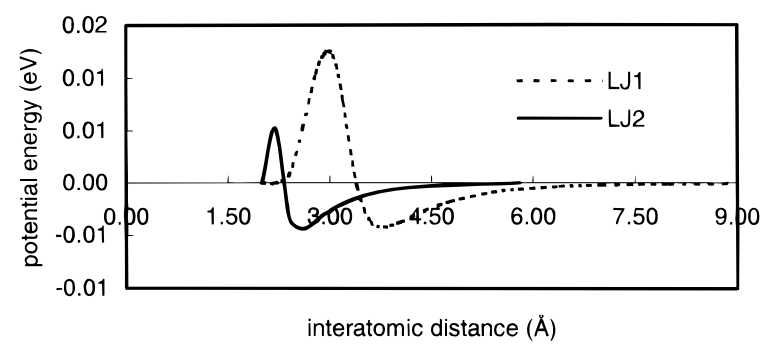

Figure 1. Long-range potential energy for $\mathrm{C}-\mathrm{C}$ interactions using LJ1 and LJ2.

time. ${ }^{32-34}$ A time step of 0.25 fs was used in all the simulations. This limits the results of the study to short time scales (ps to $\mathrm{ns}$ ). The forces on the atoms were calculated using methods that vary with distance: short-range interactions were calculated using a reactive, empirical bonding-order hydrocarbon potential that realistically describes covalent bonding within both the molecules and the carbon nanotubes. This potential was originally parametrized by Brenner to examine the growth of diamond thin films by chemical vapor deposition. ${ }^{35}$ It has also been successfully used to study reactions at surfaces ${ }^{36,37}$ and the structure and mechanical properties of carbon nanotubules..$^{38-42}$

The long-range interactions were characterized with two different Lennard-Jones ( $\mathrm{LJ})$ potentials, one that was formulated for the study of liquid $n$-butane near its boiling point, ${ }^{43}$ termed LJ1, and a $\mathrm{LJ}$ potential for generic hydrocarbon systems, ${ }^{22}$ referred to here as LJ2. The combined expression used to calculate the energy of the system in each case is

$$
U=\sum_{i} \sum_{i<j}\left[V_{\mathrm{r}}\left(r_{i j}\right)-B_{i j} V_{\mathrm{a}}\left(r_{i j}\right)+V_{v d w}\left(r_{i j}\right)\right]
$$

where $U$ is the binding energy, $r_{i j}$ is the distance between atoms $i$ and $j, V_{\mathrm{r}}$ is a pair-additive term that models the interatomic core-core repulsive interactions, and $V_{\mathrm{a}}$ is a pairadditive term that models the attractive interactions due to the valence electrons. In addition, $B_{i j}$ is a many-body empirical bond-order term that modulates valence electron densities and depends on atomic coordination and bond angles. Finally, $V_{v d w}$ is the contribution from the LJ potential and is only nonzero after the short-range covalent potential goes to zero. Figure 1 compares the long-range potential energy between two carbon atoms as calculated with LJ1 and LJ2. The most significant difference occurs in the range 2.0-4.2 $\AA$ (the average spacing between molecules in the simulations is $3.9 \AA$ ). Complete details for the way in which each $V_{v d w}$ was evaluated can be found in ref 44 .

During equilibration, $90 \%$ of the atoms in the system (molecules and nanotube wall atoms) had a Langevin thermostat $^{32}$ applied to them. During dynamic flow simulations, the equilibrated molecules were placed just inside the nanotube opening and given hyperthermal velocities in the direction of the nanotube axis. All the atoms in the nanotube walls, except where noted, were allowed to move in response to applied forces according to Newton's equations and with the additional constraint of applied Langevin frictional forces. Periodic boundary conditions ${ }^{32}$ were applied to the nanotubes (and hence also to the molecules inside the nanotubes) in the direction of the nanotube axis, which varied in length between 50 and $54 \AA$. To model molecular diffusive flow, $80 \AA$ A-long, single-walled nanotubes were used where the treatment of the atoms in the nanotube walls was the same as in the case of dynamic flow except that no periodic boundary conditions were used. The opened nanotubes were terminated with either carbon or hydrogen atoms. The molecules of interest were then placed near the opening at one end (some slightly inside the nanotube, some well outside the opening), and the system was allowed to evolve in time with no additional constraints. These starting conditions therefore correspond to an external molecular pressure gradient. We also considered pore-pore correlation effects by examining diffusion through a small close-packed bundle of $(10,0)$ nanotubes. The shortest distance between the nanotube walls in the bundle was $3.4 \AA$.

\section{Results and Discussion}

III. A. Dynamic Flow. The dynamic flow of three different types of molecules-methane, ethane, and ethylene-through single-walled carbon nanotubes with diameters of about $7 \AA$ to $36 \AA$ was considered. The helical symmetry of the nanotubes followed either the armchair form $(n, n)$ or the zigzag form $(n, 0) .{ }^{45}$ Because the potential of interaction between the atoms is conservative, the total energy should be conserved subject to changes in the nonbonding interaction between the nanotube and the molecules. The average velocity of the molecules was determined as follows:

$$
\begin{gathered}
v_{i}=\left(\frac{m_{\mathrm{C}}}{m} \sum v_{\mathrm{C}}^{2}+\frac{m_{\mathrm{H}}}{m} \sum v_{\mathrm{H}}^{2}\right)^{1 / 2} \\
v=v_{\mathrm{avg}}=\sum_{i} v_{i} / N
\end{gathered}
$$

In these equations, $v_{i}$ is the velocity of each individual molecule in the nanotubes, $m_{\mathrm{C}}$ is the atomic weight of carbon, $m_{\mathrm{H}}$ is the atomic weight of hydrogen, $m$ is the total molecular weight, $v_{\mathrm{C}}$ is the velocity of the carbon atoms in each molecule, $v_{\mathrm{H}}$ is the velocity of the hydrogen atoms in each molecule, and $N$ is the total number of molecules.

In the dynamic flow studies, the molecules were given only an incidental velocity in the direction of the nanotube axis. However, as the molecules flowed through the nanotubes, the ordered motion was transformed into random thermal vibrational motion as the molecule-nanotube interactions slowed the molecular motion. Because of the relatively high initial velocities, the majority of the molecular kinetic energy was translational. However, as the molecular motion became increasingly randomized, more kinetic energy was transformed into vibrational and rotational motion, with the latter exhibited in small "wagging" motions.

First, the flow of methane through nanotubes of different diameter was considered where the initial velocities of all the molecules inside the nanotubes were the same. The density of methane within the nanotube was $0.353 \mathrm{~g} / \mathrm{cm}^{3}(83.3 \%$ liquid density at $110 \mathrm{~K}) .{ }^{46}$ Table 1 summarizes the nanotube diameters considered and the results of the simulations for initial hyperthermal velocities of $0.1 \AA / f s$. The dynamic molecular flow decreased to thermal velocities in a few ps when the nanotube diameters were less than $36 \AA$. At nanotube diameters of $36 \AA$, there was little disruption of the dynamic flow and the molecular motion did not slow over the time scales considered. To explain this result, the potential equation of interaction between the molecules and the nanotubes, $U_{\mathrm{m}-\mathrm{t}}$, was calculated as follows:

$$
U_{\mathrm{m}-\mathrm{t}}=U_{\mathrm{tot}}-U_{\text {nanotube }}-\sum U_{\mathrm{m}-\mathrm{m}}
$$

where $U_{\mathrm{m}-\mathrm{m}}$ is the potential of interaction between molecules inside the tubules, $U_{\text {tot }}$ is the total potential energy of the system, and $U_{\text {nanotube }}$ is the potential energy of the tubule. Figure 2 
TABLE 1: Summary of Data from Simulations of Dynamic Fluid Flow of Methane Through Nanotubes of Different Diameters $^{a}$

\begin{tabular}{|c|c|c|c|c|}
\hline $\begin{array}{l}\text { diameter } \\
(\AA)\end{array}$ & $\begin{array}{l}\text { tubule } \\
\text { type }\end{array}$ & $\begin{array}{l}\text { total } \\
\text { time } \\
\text { (ps) }\end{array}$ & $\begin{array}{l}\text { distance } \\
\text { traveled } \\
(\AA)\end{array}$ & outcome \\
\hline 7.1 & $(5,5)$ & 1.6 & $\begin{array}{l}80 \\
90\end{array}$ & $\begin{array}{l}\text { slowed in } 1.5 \mathrm{ps}(\mathrm{LJ} 1) \\
\text { slowed in } 1.6 \mathrm{ps}(\mathrm{LJ} 2)\end{array}$ \\
\hline 8.0 & $(10,0)$ & 3.0 & $\begin{array}{l}120 \\
130\end{array}$ & $\begin{array}{l}\text { slowed in } 2.0 \mathrm{ps}(\mathrm{LJ} 1) \\
\text { slowed in } 2.1 \mathrm{ps}(\mathrm{LJ} 2)\end{array}$ \\
\hline 11.0 & $(8,8)$ & 11.5 & $\begin{array}{l}200 \\
205\end{array}$ & $\begin{array}{l}\text { slowed in } 4.0 \mathrm{ps}(\mathrm{LJ} 1) \\
\text { slowed in } 4.0 \mathrm{ps}(\mathrm{LJ} 2)\end{array}$ \\
\hline 16.5 & $(12,12)$ & 11.5 & $\begin{array}{l}350 \\
320\end{array}$ & $\begin{array}{l}\text { slowed in } 7.5 \text { ps to } 0.01 \AA / f s(\mathrm{LJ} 1) \\
\text { slowed in } 7.4 \text { ps to } 0.01 \AA / \mathrm{fs}(\mathrm{LJ} 2)\end{array}$ \\
\hline 25.0 & $(16,16)$ & 11.5 & $\begin{array}{l}700 \\
660\end{array}$ & $\begin{array}{l}\text { slowed in } 11.5 \text { ps to } 0.01 \AA / \mathrm{fs} \text { (LJ1) } \\
\text { slowed in } 11.0 \mathrm{ps} \text { to } 0.01 \AA / \mathrm{fs}(\mathrm{LJ} 2)\end{array}$ \\
\hline 36.0 & $(25,25)$ & 15.0 & & velocity constant (LJ1) \& (LJ2) \\
\hline
\end{tabular}

${ }^{a}$ The methane has an initial hyperthermal velocity of $0.1 \AA /$ fs in each case. The $(5,5)$ nanotube system has 625 atoms, the $(10,0)$ nanotube system has 700 atoms, the $(8,8)$ nanotube system has 800 atoms, the $(12,12)$ nanotube system has 875 atoms, the $(16,16)$ nanotube system has 940 atoms, and the $(25,25)$ nanotube system has 1050 atoms.

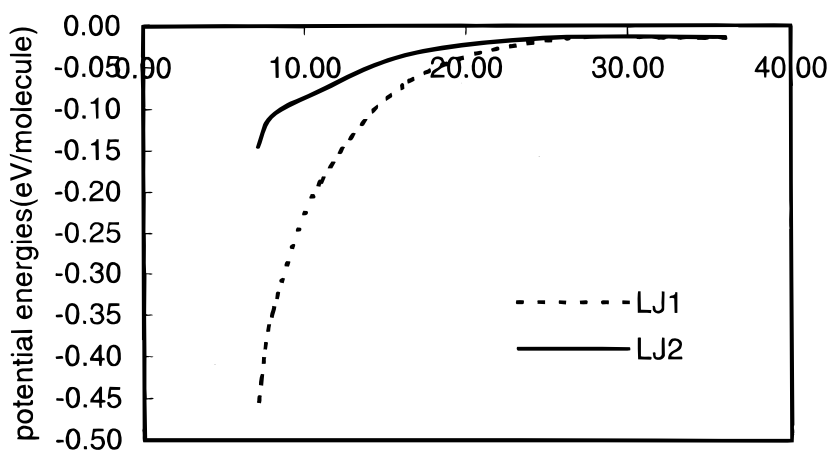

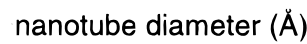

Figure 2. Nanotube-molecule interaction energy for methane in $(n, 0)$ single-walled nanotubes as calculated with LJ1 and LJ2.

illustrates how the potential of interaction between the molecules and the nanotubes decreases with increasing diameter of the nanotubes. Comparison of the results in Table 1 and Figure 2 indicates that at smaller nanotube diameters, the interactions between the molecules and the nanotubes were stronger, thus slowing the dynamic flow of the molecules more quickly than in larger nanotubes. The average distance the molecules in the larger nanotubes traveled is longer than the distance traveled in smaller nanotubes. Careful analysis of the simulation results also shows that when the nanotube diameter was less than the cutoff value of $36 \AA$, the molecules collided more frequently with the nanotube walls. Conversely, the molecule-nanotube interactions were weaker at larger nanotube diameters, so the molecules flowed relatively freely through the nanotube with little change in velocity over time. Finally, the results showed little dependence on the way in which the van der Waals interaction energy was determined.

Next, the dynamic flow of methane molecules through a $(10,0)$ tubule with an $8.0 \AA$ diameter at different initial velocities of $0.05,0.1,0.3$, and $0.5 \AA / f$ s was considered. The simulations used only LJ1, and the results are summarized in Figure 3. The figure shows that after about $2.0 \mathrm{ps}$, the velocities of all the molecules were reduced to thermal velocities. At higher dynamic velocities the molecules slowed more rapidly than at lower velocities, all other factors being equal.

The next series of simulations examined the dynamic flow of ethane and ethylene. The densities were $0.408 \mathrm{~g} / \mathrm{cm}^{3}$ for ethane and $0.386 \mathrm{~g} / \mathrm{cm}^{3}$ for ethylene. ${ }^{47}$ The hyperthermal

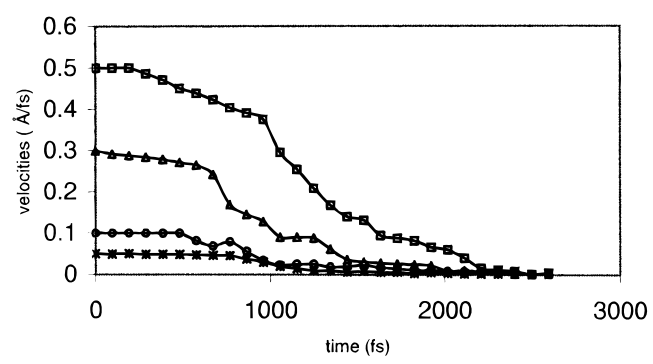

Figure 3. Results for dynamic flow of methane starting from various initial velocities in a $(10,0)$ nanotube.

TABLE 2: Summary of Data from Simulations of Dynamic Flow of Various Fluid Molecules in $(10,0)$ Nanotubes $8.0 \AA$ in Diameter ${ }^{a}$

\begin{tabular}{|c|c|c|c|c|}
\hline $\begin{array}{l}\text { molecule } \\
\text { type }\end{array}$ & $\begin{array}{l}\text { velocity } \\
(\AA / \mathrm{fs})\end{array}$ & $\begin{array}{l}\text { total } \\
\text { time } \\
(\mathrm{ps})\end{array}$ & $\begin{array}{c}\text { distance } \\
\text { traveled } \\
(\AA)\end{array}$ & outcome \\
\hline methane & 0.1 & 3.0 & $\begin{array}{l}120 \\
130\end{array}$ & $\begin{array}{l}\text { slowed in } 2.0 \mathrm{ps}(\mathrm{LJ} 1) \\
\text { slowed in } 2.1 \mathrm{ps}(\mathrm{LJ} 2)\end{array}$ \\
\hline methane & 0.5 & 4.0 & 780 & slowed in $2.7 \AA \mathrm{ps}(\mathrm{LJ} 1)$ \\
\hline ethane & 0.1 & 2.0 & $\begin{array}{l}78 \\
70\end{array}$ & $\begin{array}{l}\text { slowed in } \sim 1.3 \mathrm{ps}(\mathrm{LJ} 1) \\
\text { slowed in } 1.4 \mathrm{ps}(\mathrm{LJ} 2)\end{array}$ \\
\hline ethane & 0.5 & 2.0 & & chemical reactions (LJ1) \\
\hline ethylene & 0.1 & 2.0 & $\begin{array}{l}78 \\
66\end{array}$ & $\begin{array}{l}\text { slowed in } \sim 1.5 \mathrm{ps}(\mathrm{LJ} 1) \\
\text { slowed in } 1.3 \mathrm{ps}(\mathrm{LJ} 2)\end{array}$ \\
\hline ethylene & 0.5 & 2.0 & & chemical reactions (LJ1) \\
\hline
\end{tabular}

${ }^{a}$ The methane system has 625 atoms, the ethane system has 1100 atoms, and the ethylene system has 1050 atoms.

dynamic flow velocities, determined using LJ1, slowed to thermal velocities in 1.5 ps for ethylene and 1.3 ps for ethane, both much shorter times than was the case with methane. For initial velocities of $0.05 \AA / \mathrm{fs}$, the dynamic flow slowed more rapidly than when the molecules had an initial velocity of 0.1 $\AA / f$ s. When the molecular velocity equaled $0.5 \AA / f$ s, the ethane or ethylene molecules collided frequently with each other or with the nanotube walls and reacted chemically to form new molecules, ions, and fragments. These frequent collisions and reactions slowed the dynamic flow by transferring some of the external molecular translational kinetic energy to internal molecular energy to overcome the barriers to reaction.

The results for dynamic flow at initial velocities of 0.1 and $0.5 \AA /$ fs as a function of molecule type are summarized in Table 2. The table shows that larger molecules slowed more rapidly than small molecules. This occurred because ethane and ethylene each have greater mass than methane, which leads to stronger nanotube-molecule interactions. This finding agrees with the results of Tuzun et al. ${ }^{30}$ for argon and helium flowing through carbon nanotubes, where the heavier argon atoms were predicted to slow more rapidly than the helium atoms. The table also summarizes the finding that dynamic flow at the highest initial velocities of $0.5 \AA / f s$ introduces the possibility of reactive molecular collisions with other molecules or the nanotube walls for ethane and ethylene.

The simulations predict that the molecular density inside the nanotubes can affect dynamic flow. Figure 4 shows the results for different densities of methane flowing through a $(10,0)$ nanotube with a diameter of $8.0 \AA$. The densities considered were $0.353,0.302$, and $0.250 \mathrm{~g} / \mathrm{cm}^{3}$. The figure shows that the higher the molecular density, the more rapidly the molecules slowed to thermal velocities. The explanation for this behavior is that as the density increases, the average distance between the molecules becomes smaller and the interactions between the molecules increase, thus slowing down the dynamic flow more rapidly. 


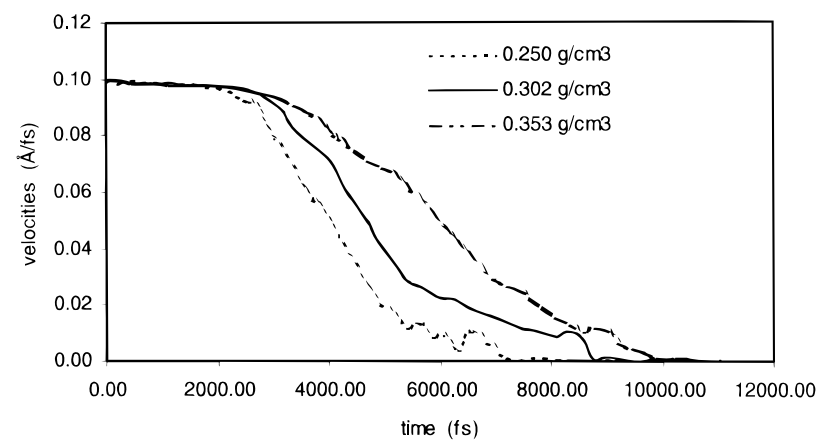

Figure 4. Dynamic flow of methane at various densities in a $(10,0)$ nanotube.

Additional simulations were performed to study the flow of methane through $8.0 \AA$-diameter $(10,0)$ nanotubes that had 10 rigid atoms at each end. The molecular velocity in these partially rigid nanotubes decreased more slowly than in the fully dynamic nanotubes. This occurs because as the nanotube moves, it perturbs the motion of the nearby molecules, thus randomizing their motion more rapidly and resulting in more molecular collisions with the nanotube walls. These findings also agree with the results of Tuzun et al. ${ }^{30}$ As the molecules moved through the dynamic nanotubes, the diameter of the nanotubes increased slightly and the nanotubes straightened. This effect became more pronounced at higher molecular velocities.

Simulations similar to those described in this section were performed with LJ2 and the results were only slightly different from the results obtained with LJ1. For the same simulation system, LJ1 slowed the molecular flow about 5\% faster than LJ2. However, the trends in all cases were the same. Hence, small differences in the LJ potential do not greatly affect simulation results for dynamic molecular flow because at the high hyperthermal velocities considered, molecule-molecule interactions have less effect than the molecule-nanotube interactions, and at larger distances, LJ1 and LJ2 are nearly the same.

III. B. Diffusive Flow. Preliminary results for the diffusion of methane, ethane, and ethylene through carbon-terminated $(10,0)$ nanotubes (diameter $=8 \AA$ ) ranging in length from 20 to $80 \AA$ are reported in ref 44 . The simulations predicted that the molecules intercalated into the nanotubes and diffused down their length from the areas of high density to the areas of low density. The methane motion followed normal-mode diffusion, which can be expressed as:

$$
S^{2}=2 A t
$$

where $S$ is the average distance that the molecules move, $A$ is the diffusion coefficient, and $t$ is time. In this diffusion mode, individual molecules can pass each other within the pore. In contrast, ethane and ethylene show diffusion behavior that is intermediate between normal-mode and single-file diffusion in the simulations. In single-file diffusion, individual molecules cannot pass each other because of their large size relative to the pore diameter. Single-file diffusion is therefore expressed as:

$$
S^{2}=2 B t^{0.5}
$$

where $B$ is the diffusion mobility. These results show good agreement with the simulation results of Keffer et al. ${ }^{16}$ for the diffusion in zeolites.

Figure 5 shows a $\log -\log$ plot of the average diffusion distance of these molecules as a function of time, where all the
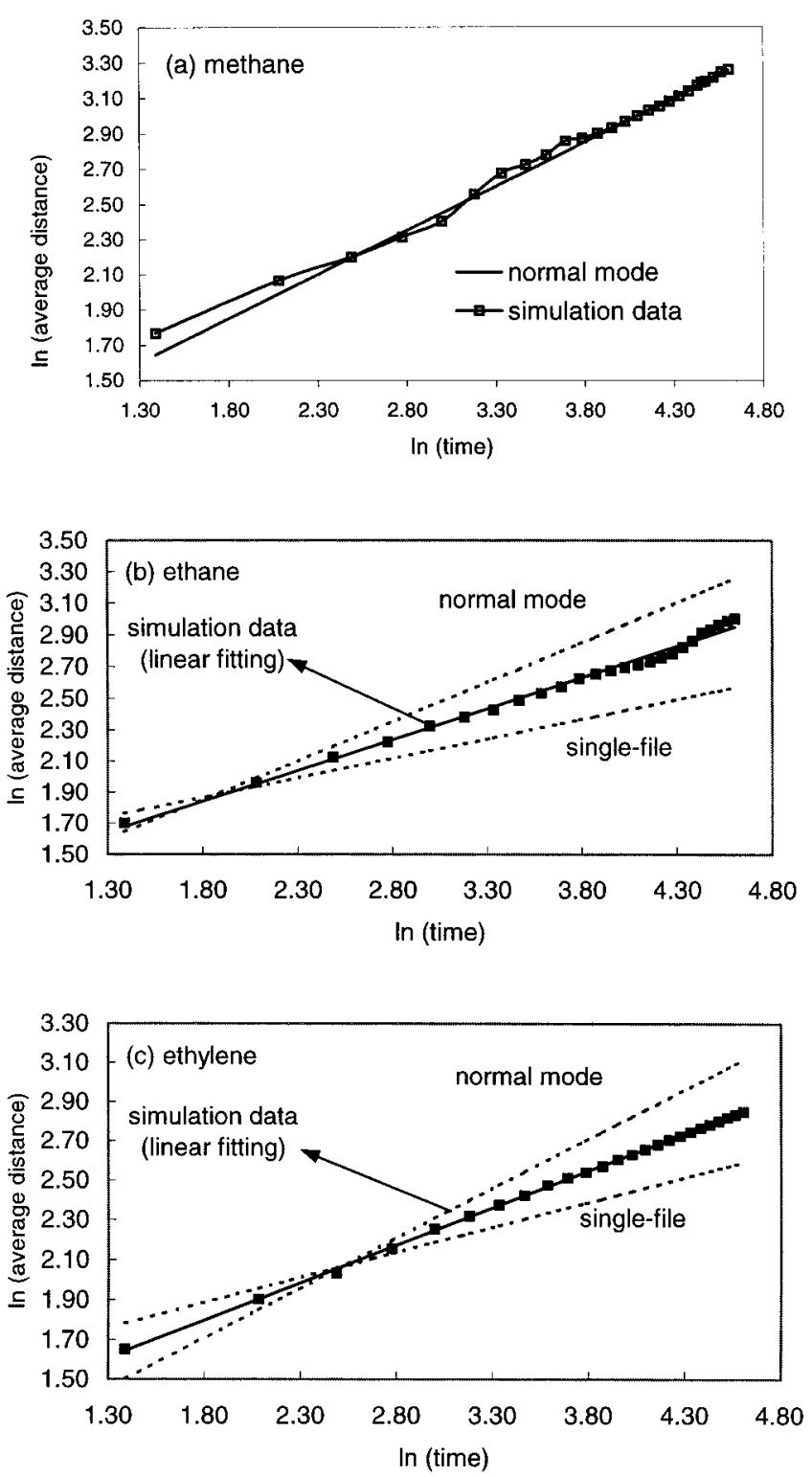

Figure 5. $\log -\log$ plot of distance vs time for the diffusive flow of (a) methane, (b) ethane, and (c) ethylene in a $(10,0)$ nanotube. LJ2 was used in these simulations.

simulations ran for $100 \mathrm{ps}$. The results indicate that if the molecular structure is spherical, as it is in methane, the diffusion behavior can be clearly distinguished as either normal-mode or single-file mode. Transition-mode diffusion was not predicted for methane because small-angle molecular rotations during diffusion had no effect on the rate at which molecules can pass each other. In nanotubes with diameters that are large enough for the methane molecules to pass each other, such as were considered in this study, methane exhibited normal-mode diffusion.

In contrast, if the molecular shape was highly asymmetrical and could not be treated as a sphere, as with ethane and ethylene, transition-mode diffusion was predicted over the short time scales of this study. This type of diffusion occurred in nanotubes with diameters large enough for the molecules to pass each other if they were all perfectly aligned parallel to the nanotube axis, but not large enough to allow molecules to pass each other if some of them had undergone small-angle rotational motion during diffusion. Thus, as ethane and ethylene diffused, the molecules were able to pass each other some of the time but not during other times. This result highlights the fact that 
TABLE 3: Summary of Data from Simulations of Diffusion of Methane, Ethane, and Ethylene in Various Zigzag Nanotubes (unit: $\mathrm{cm}^{2} / \mathrm{s}$ for normal mode and $\mathrm{cm}^{2} / \mathrm{s}^{0.5}$ for single-file mode) ${ }^{a}$

\begin{tabular}{cllllllll}
\hline type & \multicolumn{1}{c}{$(9,0)$} & \multicolumn{1}{c}{$(10,0)$} & \multicolumn{1}{c}{$(12,0)$} & \multicolumn{1}{c}{$(14,0)$} & \multicolumn{1}{c}{$(16,0)$} & $(18,0)$ & $(20,0)$ & $(22,0)$ \\
\hline diameter & $7.2 \AA$ & $8.0 \AA$ & $9.53 \AA$ & $11.1 \AA$ & $12.7 \AA$ & $14.3 \AA$ & $15.9 \AA$ & $17.5 \AA$ \\
\hline methane & normal & normal & normal & normal & normal & normal & normal & normal \\
& $3.91 \times 10^{-4}$ & $3.80 \times 10^{-4}$ & $2.94 \times 10^{-4}$ & $1.86 \times 10^{-4}$ & $1.05 \times 10^{-4}$ & $8.32 \times 10^{-5}$ & $4.25 \times 10^{-5}$ & $1.64 \times 10^{-5}$ \\
& $(2600)$ & $(2700)$ & $(2975)$ & $(3150)$ & $(3325)$ & $(3500)$ & $(3620)$ & $(3700)$ \\
ethane & single-file & transition & transition & transition & transition & normal & normal & normal \\
& $9.65 \times 10^{-10}$ & $(3150)$ & $(3450)$ & $(3580)$ & $(3660)$ & $9.65 \times 10^{-5}$ & $6.85 \times 10^{-5}$ & $2.92 \times 10^{-5}$ \\
& $(2850)$ & & & & & $(3850)$ & $(3950)$ & $(4250)$ \\
ethylene & single-file & transition & transition & transition & normal & normal & normal & normal \\
& $9.15 \times 10^{-10}$ & $(3050)$ & $(3350)$ & $(3480)$ & $9.81 \times 10^{-5}$ & $8.48 \times 10^{-5}$ & $6.35 \times 10^{-5}$ & $244 \times 10^{-5}$ \\
& $(2750)$ & & & & $(3540)$ & $(3720)$ & $(3820)$ & $(4120)$
\end{tabular}

${ }^{a}$ The number in parentheses is the number of atoms in the system.

TABLE 4: Summary of How the Transitional Diffusion Mode of Ethane and Ethylene in Different-Size Nanotubes Changes with Nanotube Diameter ${ }^{a}$

\begin{tabular}{|c|c|c|c|c|c|c|}
\hline tube type & \multicolumn{2}{|c|}{$(10,0)$} & \multicolumn{2}{|c|}{$(12,0)$} & \multicolumn{2}{|c|}{$(14,0)$} \\
\hline diameter & \multicolumn{2}{|c|}{$8.00 \AA$} & \multicolumn{2}{|c|}{$9.53 \AA$} & \multicolumn{2}{|c|}{$11.1 \AA$} \\
\hline$n$ & \multicolumn{2}{|c|}{$\begin{array}{c}0.74 \\
\mathrm{~cm}^{2} / \mathrm{s} 0.74\end{array}$} & \multicolumn{2}{|c|}{0.85} & \multicolumn{2}{|c|}{0.92} \\
\hline units & \multicolumn{2}{|c|}{$\begin{array}{c}\mathrm{cm}^{2} / \mathrm{s}^{0.74} \\
3150 \text { for ethane }\end{array}$} & \multicolumn{2}{|c|}{$\mathrm{cm}^{2} / \mathrm{s}^{0.85}$} & \multicolumn{2}{|c|}{$\mathrm{cm}^{2} / \mathrm{s}^{0.92}$} \\
\hline \# atoms & \multirow{2}{*}{\multicolumn{2}{|c|}{$\begin{array}{l}3150 \text { for ethane } \\
3050 \text { for ethylene }\end{array}$}} & \multirow{2}{*}{\multicolumn{2}{|c|}{$\begin{array}{c}3450 \text { for ethane } \\
3350 \text { for ethylene }\end{array}$}} & \multirow{2}{*}{\multicolumn{2}{|c|}{$\begin{array}{l}3580 \text { for ethane } \\
3480 \text { for ethylene }\end{array}$}} \\
\hline & & & & & & \\
\hline C (ethane) & $\begin{array}{l}5.44 \times 10^{-8} \\
(\mathrm{LJ} 1)\end{array}$ & $\begin{array}{l}2.45 \times 10^{-8} \\
(\mathrm{LJ} 2)\end{array}$ & $\begin{array}{l}8.15 \times 10^{-7} \\
(\mathrm{LJ} 1)\end{array}$ & $\begin{array}{l}3.20 \times 10^{-7} \\
(\mathrm{LJ} 2)\end{array}$ & $\begin{array}{l}7.39 \times 10^{-6} \\
(\mathrm{LJ} 1)\end{array}$ & $\begin{array}{l}3.72 \times 10^{-6} \\
(\text { LJ2) }\end{array}$ \\
\hline C (ethylene) & $\begin{array}{l}5.25 \times 10^{-8} \\
(\mathrm{LJ} 1)\end{array}$ & $\begin{array}{l}1.96 \times 10^{-8} \\
(\mathrm{LJ} 2)\end{array}$ & $\begin{array}{l}8.06 \times 10^{-7} \\
(\mathrm{LJ} 1)\end{array}$ & $\begin{array}{l}3.05 \times 10^{-7} \\
(\mathrm{LJ} 2)\end{array}$ & $\begin{array}{l}7.24 \times 10^{-6} \\
(\mathrm{LJ} 1)\end{array}$ & $\begin{array}{l}3.55 \times 10^{-6} \\
(\mathrm{LJ} 2)\end{array}$ \\
\hline
\end{tabular}

${ }^{a}$ The listed parameters are for eq 6 . The data were obtained from simulations that ran $100 \mathrm{ps}$ for the $(10,0)$ nanotubes and $40 \mathrm{ps}$ for the $(12,0)$ and $(14,0)$ nanotubes.

nanopore-diffusion simulations that approximate ethane or ethylene as spheres will not predict this behavior. We have developed a general equation to quantify the description of the transition-mode diffusion of ethane and ethylene as follows:

$$
S^{2}=2 C t^{n}
$$

where $C$ is the diffusion mobility and $n$ is a coefficient that depends on molecular type and pore diameter. The index $n$ reflects the rate at which molecules can pass each other and provides an indication of the small-angle molecular rotation.

Table 3 summarizes the effect of nanotube size on the diffusion behavior of ethane and ethylene, using LJ1 for nanotubes with the same helical configuration. From the results it can be seen that as the nanotube diameter increased, the diffusion modes of the larger molecules changed from singlefile to normal-mode. This change occurred because as the size of the nanotube increased, the molecules were able to pass each other more easily. Thus, the nanotube diameters in which ethane and ethylene exhibited transition-mode diffusion varied over a relatively small range from $8.0 \AA$ to 11.0 or $12.7 \AA$. In the case of the $(25,25)$ nanotube, with a diameter of $36 \AA$, diffusion was not observed on the time scales of these classical molecular dynamics simulations. It should be noted that $36 \AA$ is the generally accepted cutoff for changes from diffusive motion to flow through other mechanisms, such as capillary motion. ${ }^{48}$ Such motion could be investigated, for example, using a combination of MD and MC, as was done in ref 17.

From the data shown in Figure 5, an $n$ value of 0.74 in eq 6 was derived for both ethane and ethylene in $(10,0)$ tubes. As the diameter of the nanotubes increased, $n$ increased, as summarized in Table 4 . The value of $n$ was 0.85 for the $(12,0)$ nanotubes and 0.92 for the $(14,0)$ nanotubes for both ethane and ethylene. Thus, the simulations showed that as the size of the nanotubes increases, the index $n$ increases and molecules can more easily pass one other.
It should be pointed out that MC and MD simulations designed to study diffusion in zeolites over significantly longer time scales ${ }^{49-51}$ found that transition-mode molecular diffusion behavior resolved itself into normal-mode diffusion over time. In addition, MD studies ${ }^{52}$ have suggested that when molecules have the ability to pass each other in the nanopore, small changes in the system can have a significant effect on the results, which further complicates the characterization of transition-mode diffusion. Temperature also has been shown to play a role in determining the diffusion mode ${ }^{53}$ where higher temperatures allow molecules that might normally not be able to, to squeeze past one another. Experimentally, no transition-mode diffusion has been observed in zeolites, but different experiments show single-file and normal-mode diffusion for the same system. ${ }^{50}$ Thus, it should be recognized that eq 6 is primarily a way to quantify the motion of nonspherical molecules predicted in these simulations, rather than a universal expression.

The effects of nanotube helical symmetry were examined by studying diffusion in similar diameter nanotubes that were either armchair or zigzag type. An example is the diffusion of methane in $(14,0)$ (radius $=5.56 \AA$ ) and $(8,8)$ (radius $=5.50 \AA$ ) nanotubes under otherwise identical conditions. In both cases normal-mode diffusion was predicted. The diffusion coefficients are $1.86 \times 10^{-4} \mathrm{~cm}^{2} / \mathrm{s}$ for the $(14,0)$ nanotube and $1.78 \times 10^{-4}$ $\mathrm{cm}^{2} / \mathrm{s}$ for the $(8,8)$ nanotube. These coefficients indicate that the helical symmetry of the nanotube has little effect on the diffusion behavior of methane. However, an interesting, previously unreported phenomenon was predicted for ethane and ethylene diffusing in nanotubes with diameters between 16 and $22 \AA$. In these nanotubes, ethane and ethylene molecules diffused following a spiral path around the circumference of the nanotube. In these cases, the paths followed by the molecules were strongly correlated to the helical structure of the specific nanotubes. For example, in one spiral cycle individual molecules move a longer distance in zigzag tubes than armchair tubes because of the differing helical structure. The driving force for 

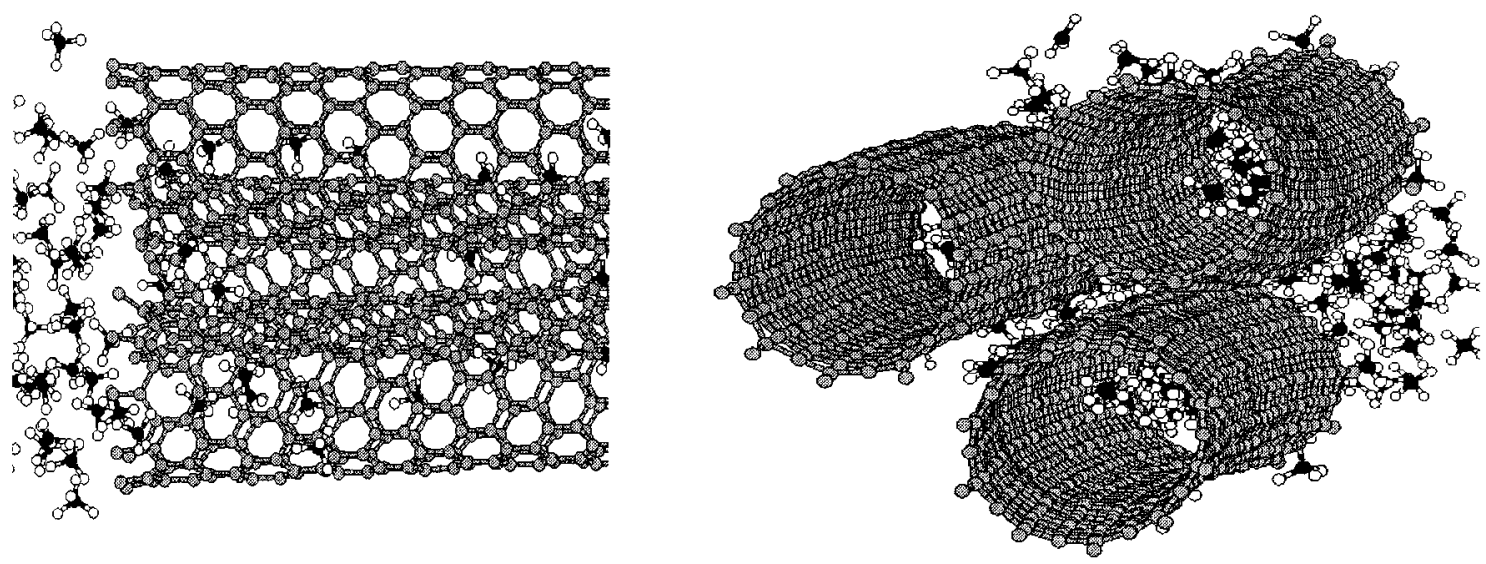

Figure 6. Snapshots from simulations of methane diffusion into $(10,0)$ carbon-terminated nanotubes arranged in a bundle.

TABLE 5: Summary of the Effects of the Potential Used to Calculate van der Waals Interactions during Molecular Diffusion in Single $(\mathbf{1 0 , 0})$ Nanotubes $80 \AA$ Long ${ }^{a}$

\begin{tabular}{lllc}
\hline \multirow{2}{*}{$\begin{array}{c}\text { molecular } \\
\text { type }\end{array}$} & $\begin{array}{c}\text { diffusion } \\
\text { mode }\end{array}$ & \multicolumn{2}{c}{ diffusion coefficients and mobilities } \\
\cline { 3 - 4 } & LJ1 & LJ2 \\
\hline methane & normal mode & $3.80 \times 10^{-4} \mathrm{~cm}^{2} / \mathrm{s}$ & $2.55 \times 10^{-4} \mathrm{~cm}^{2} / \mathrm{s}$ \\
ethane & transition mode & $5.44 \times 10^{-8} \mathrm{~cm}^{2} / \mathrm{s}^{0.74}$ & $2.45 \times 10^{-8} \mathrm{~cm}^{2} / \mathrm{s}^{0.74}$ \\
ethylene & transition mode & $5.25 \times 10^{8} \mathrm{~cm}^{2} / \mathrm{s}^{0.74}$ & $1.96 \times 10^{-8} \mathrm{~cm}^{2} / \mathrm{s}^{0.74}$ \\
\multicolumn{3}{c}{${ }^{a}$ The simulation time was $100 \mathrm{ps}}$.
\end{tabular}

this spiral diffusion path was that the interaction energy between ethane or ethylene and the nanotube wall was maximized when the molecules lined up with the carbon-carbon bonds in the nanotube wall. To maintain this high level of interaction energy, the molecules moved forward by aligning with neighboring carbon-carbon bonds within the nanotube wall, which led to the helical path. Some experimental and first-principles evidence exists that similar behavior occurs for $\mathrm{I}_{3}{ }^{-}$and $\mathrm{I}_{5}{ }^{-}$intercalating in $(10,10)$ tubes. ${ }^{54}$ Further details of this phenomenon will be discussed in a future paper.

The effect of the method used to calculate the van der Waals interactions on diffusion has also been examined. Larger differences in diffusion coefficients were predicted than were seen in the dynamic flow velocities. These differences are summarized in Table 5. In general, diffusion coefficients or mobilities calculated using LJ1 are about 1.5-2.5 times higher than those calculated using LJ2. These findings are explained by the stronger LJ1 interactions as compared to LJ2 in the interaction regions that are important for diffusion (see Figures 1 and 2). However, the overall qualitative behavior of the diffusion motion, and the diffusion mode that was followed, was unchanged between the two methods of calculating the van der Waals interactions.

We next considered the effect of atomic termination at the nanotube opening on the diffusion results. Instead of bare carbon at the opening, each edge carbon atom was terminated with a hydrogen atom. The results showed that the nanotube termination had a significant effect on the diffusion for methane. In the limit of low densities of methane $\left(0.110 \mathrm{~g} \mathrm{~cm}^{-3}\right)$, the molecules did not diffuse into the nanotubes during the simulations (100 ps). However, as the methane density increased, the effect of atomic termination on the diffusion results decreased. For example, when the methane density was 0.353 $\mathrm{g} \mathrm{cm}^{-3}$, the diffusion coefficient was about two-thirds of the value for diffusion into carbon-terminated nanotubes. Interest- ingly, in the case of ethane and ethylene, there was little change in the diffusion behavior between the carbon-terminated and hydrogen-terminated cases, because the smaller methane molecules were more sensitive to the decreased attraction at the opening of the nanotube caused by hydrogen termination. For the larger ethane and ethylene molecules, the hydrogenterminated edges were not as significant as the stronger molecule-nanotube wall interactions.

The last series of simulations considered the effect of porepore correlations on the diffusion results by examining the diffusive flow of methane through a carbon-terminated nanotube bundle, as illustrated in Figure 6, and a hydrogen-terminated nanotube bundle (not shown). Both these nanotube bundles consisted of $(10,0)$ nanotubes with diameters of $8 \AA$. The simulations were performed under the same conditions as the studies with single nanotubes discussed above. The simulations show diffusion into the nanotubes but not into the interstitial sites between the nanotubes that are about $4 \AA$ in diameter. This behavior can be explained by examining the interaction energy in these spaces: the energy of a methane molecule inside one of the nanotubes is $-0.24 \mathrm{eV} /$ atom, whereas its energy in the interstitial site between the nanotubes is $-0.16 \mathrm{eV} /$ atom. However, it is expected that the space between the nanotubes will become larger and that interactions between them will decrease as the nanotube diameters increase. These changes will make molecular intercalation into the channels more energetically favorable.

Compared to the diffusion in a single $(10,0)$ nanotube, the diffusion velocity through the bundle nanotubes was slightly decreased. This decrease occurred because the nanotubenanotube (pore-pore) interactions decreased the moleculenanotube-wall interactions. The diffusion mode for methane was still normal-mode in all the cases considered. The diffusion coefficient was $2.57 \times 10^{-4} \mathrm{~cm}^{2} / \mathrm{s}$ as calculated with LJ1 and $5.65 \times 10^{-5} \mathrm{~cm}^{2} / \mathrm{s}$ as calculated with $\mathrm{LJ} 2$ for the carbonterminated case. In the hydrogen-terminated case, the diffusion coefficient was $2.14 \times 10^{-4} \mathrm{~cm}^{2} / \mathrm{s}$ as calculated with LJ1 and $4.85 \times 10^{-5} \mathrm{~cm}^{2} / \mathrm{s}$ as calculated with $\mathrm{LJ} 2$.

\section{Conclusions}

Atomistic simulations have been used to study the flow of molecules inside carbon nanotubes at room temperature. During studies of dynamic flow, several factors were predicted to affect the flow velocity over time. The first is the size (diameter) of the nanotubes. As the diameter of the carbon nanotubes 
decreased, the dynamic flow of the fluid molecules slowed more rapidly. The molecular density was also shown to have an effect. At higher fluid densities, the velocity of the molecules slowed more quickly because of increased interactions between the molecules. Next, the effect of molecular type was considered. Ethane and ethylene molecules slowed more rapidly than methane because they have stronger interactions with the nanotube walls. Finally, the rigidity of the nanotube walls affected the dynamic molecular flow. Completely dynamic (nonrigid) nanotubes slowed fluids more rapidly than partially rigid nanotubes because the dynamic motion of the nanotube walls disturbed the flow of the molecules to a greater extent than the partially rigid nanotube walls. This caused more collisions between the molecules and the nanotube walls. The motion of the molecules also tended to make the nanotubes expand and straighten.

The diffusive flow of methane, ethane, and ethylene molecules through nanotubes was also investigated at room temperature. The hydrogen-terminated edge of the nanotube had a significant effect on methane but almost no effect on ethane and ethylene. Pore-pore correlations caused the diffusion velocities and the coefficients and mobilities to decrease. The size of the nanotubes had a significant effect on the molecular diffusion, with the diffusion mechanism changing as the diameter of the nanotubes increased. At diameters of $36 \AA$, no diffusion was observed on the short time scales of the MD simulations. The diffusion mode for ethane and ethylene in nanotubes with diameters between about 8 and $12 \AA$ was transitional between single-file and normal-mode diffusion. A model was developed to quantify this behavior. However, other studies in the literature suggest that at longer times these transitional diffusion modes approach normal-mode. In nanotubes with diameters between 16 and $22 \AA$, ethane and ethylene were predicted to follow a previously unreported helical diffusion path to maximize the energy of interaction between the carbon-carbon molecular bonds and the carbon-carbon bonds in the nanotube walls.

Two methods, denoted as LJ1 and LJ2, were used to calculate the van der Waals interactions among the molecules and between the molecules and the nanotube walls. The differences between these methods had little effect on the dynamic flow of molecules through the nanotubes. However, they played a significant role in the diffusion studies. The diffusion coefficients and mobilities of methane, ethane, and ethylene at room temperature as calculated with LJ1 were about 1.5-2.5 times larger than values calculated with LJ2. This result indicates that for molecular diffusion, the method used to calculate the van der Waals interactions plays an important role in determining diffusion coefficients and mobilities.

Acknowledgment. The authors thank E. Grulke for many helpful discussions and gratefully acknowledge support from the NASA-Ames Research Center (NAG 20-1121) and the National Science Foundation MRSEC at the University of Kentucky (DMR-9809686).

\section{References and Notes}

(1) Iijima, S. Nature 1991, 56, 354

(2) Caruana, C. M. Chem. Eng. Prog. 1997, 17

(3) Cheryan, M. Ultrafiltration Membranes; Technomic Publishing Company: PA, 1986, p 279.

(4) Dillon, A. C.; Jones, K. M.; Bekkedahl, T. A.; Kiang, C. H.; Bethune, D. S.; Heben, M. J. Nature 1997, 386, 377.

(5) Sloan, J.; Hammer, J.; Zwiefka-Sibley, M.; Green, M. L. H. Chem. Commun. 1998, 3, 347.

(6) Liu, J.; Rinzler, A. G.; Dai, H.; Hafner, J. H.; Bradley, R. K.; Boul, B. J.; Lu, A.; Iverson, T.; Shelimov, K.; Huffman, C. H.; Rodriguez-Macias, F.; Shon, Y.-S.; Lee, T. R.; Colbert, D. T.; Smalley, R. E. Science 1998, 280, 1253.
(7) Karger, J.; Ruthven, D. M. Diffusion in Zeolites and Microporous Solids; Wiley and Sons: New York, 1992.

(8) Carberry, J. J. Chemical and Catalytic Reaction Engineering; McGraw-Hill: New York, 1976.

(9) de Vos, R. M.; Verweij, H. Science 1998, 279, 1710. 193.

(10) Cracknell, R. F.; Nicholson, D.; Quirke, N. Mol. Phys. 1994, 13,

(11) Bouyermaouen, A.; Bellemans, A. J. Chem. Phys. 1998, 108, 2170.

(12) Xu, L.; Tsotsis, T. T.; Sahimi, M. J. Chem. Phys. 1999, 111, 3252.

(13) Gergidis, L. N.; Theodorou, D. N. J. Phys. Chem. B 1999, 103, 3380.

(14) See, for example, Kukla, V.; Kornatowski, J.; Demuth, D.; Girnus, I.; Pfeifer, H.; Rees, L. V. C.; Schunk, S.; Unger, K. K.; Karger, J. Science 1996, $272,702$.

(15) See, for example, Keffer, D.; Davis, H. T.; McCormick, A. V. Adsorption 1996, 2, 9.

(16) Keffer, D.; McCormick, A. V.; Davis, H. T. Mol. Phys. 1996, 87 , 367.

(17) Sholl, D. S.; Fichthorn, K. A. J. Chem. Phys. 1997, 107, 4384.

(18) Sastre, G.; Catlow, C. R. A.; Corma, A. J. Phys. Chem. B 1999, $103,5187$.

(19) Mosell, T.; Schrimpf, G.; Brichmann, J. J. Phys. Chem. B 1997, 101, 9476; 1997, 101, 9485 .

(20) Gladden, L. F.; Sousa-Goncalves, J. A.; Alexander, P. J. Phys. Chem. B 1997, 101, 10121

(21) Webb III, E. B.; Grest, G. S.; Mondello, M. J. Phys. Chem. B 1999, $103,4949$.

(22) Saravanan, C.; Auerbach, S. M. J. Chem. Phys. 1999, 110, 11000.

(23) Ebbesen, T. W. J. Phys. Chem. Solids 1996, 57, 951.

(24) Eswaramoorthy, M.; Sen, R.; Rao, C. N. R. Chem. Phys. Lett. 1999, 304, 207.

(25) Ugarte, D.; Chatelain, A.; de Heer, W. A. Science 1996, 274, 1897.

(26) Pederson, M. R.; Broughton, J. Q. Phys. Rev. Lett. 1992, 69, 2689

(27) Darkrim, F.; Levesque, D. J. Chem. Phys. 1998, 109, 4981.

(28) Wang, Q.; Johnson, J. K. J. J. Chem. Phys. 1999, 110, 577. Wang, Q.; Johnson, J. K. J. Phys. Chem. B 1999, 103, 4809.

(29) Dresselhaus, M. S.; Williams, K. A.; Eklund, P. C. MRS Bull. 1999, 24,45

(30) Tuzun, R. E.; Noid, D. W.; Sumpter, B. G.; Merkle, R. C. Nanotechnology 1996, 7, 241.

(31) Wang, Q.; Challa, S. R.; Sholl, D. S.; Johnson, J. K. Phys. Rev. Lett. 1999, 82, 956

(32) Allen, M. P.; Tildesley, D. J. Computer Simulation of Liquids; Oxford University Press: New York, 1987.

(33) Gear, C. W. Numerical Integration of Ordinary Differential Equations of Various Orders; Report ANL 7126, Argonne National Laboratory: Argonne, IL, 1966.

(34) Gear, C. W. Numerical Initial Value Problems in Ordinary Differential Equations: Prentice Hall: Englewood Cliffs, NJ, 1971.

(35) Sinnott, S. B.; Qi, L.; Shenderova, O. A.; Brenner, D. W. In Advances in Classical Trajectory Methods, Molecular Dynamics of Clusters, Surfaces, Liquids, and Interfaces; Hase, W., Ed.; JAI Press: Stamford, CT, 1999; Volume IV, Chapter 1, pp 1-26.

(36) Williams, E. R.; Jones, G. C., Jr.; Fang, L.; Zare, R. N.; Garrison, B. J.; Brenner, D. W. J. Am. Chem. Soc. 1992, 114, 3207.

(37) Qi, L.; Sinnott, S. B. J. Vac. Sci. Technol., A 1998, 16, 1293.

(38) Yakobson, B. I.; Brabec, C. J.; Bernholc, J. Phys. Rev. Lett. 1996, 76, 2511.

(39) Garg, A.; Han, J.; Sinnott, S. B. Phys. Rev. Lett. 1998, 81, 2260. Garg, A.; Sinnott, S. B. Chem. Phys. Lett. 1998, 295, 273.

(40) Cornwell, C. F.; Wille, L. T. Solid State Commun. 1997, 101, 555.

(41) Sinnott, S. B.; Shenderova, O. A.; White, C. T.; Brenner, D. W. Carbon 1998, 36, 1.

(42) Robertson, D. H.; Brenner, D. W.; White, C. T. J. Phys. Chem. 1992, 96, 6133

(43) Ryckaert, J.-P.; Bellemans, A. Chem. Phys. Lett. 1975, 30, 123.

(44) Mao, Z.; Garg, A.; Sinnott, S. B. Nanotechnology 1999, 10, 273.

(45) Dresselhaus, M. S.; Dresselhaus, G.; Eklund, P. C. Science of Fullerenes and Carbon Nanotubes; Academic Press: San Diego, 1996.

(46) The normal density of methane liquid at $110 \mathrm{~K}$ is $0.424 \mathrm{~g} / \mathrm{cm}^{3}$.

(47) The normal density of liquid ethane is $0.456 \mathrm{~g} / \mathrm{cm}^{3}$; of liquid ethylene, $0.570 \mathrm{~g} / \mathrm{cm}^{3}$.

(48) Morooka, S.; Kusakabe, K. MRS Bull. 1999, 24, number 3, 25

(49) Nelson, P. H.; Scott, M. A. J. Chem. Phys. 1999, 100, 9235.

(50) Cuthbert, T. R.; Wagner, N. J.; Paulaitis, M. E.; Murgia, G.; D’Aguanno, B. Macromolecules 1999, 32, 5017.

(51) See Sholl, D. S. Chem. Eng. J. 1999, 74, 25, and references therein

(52) Hahn, K. and Karger, J. J. Phys. Chem. B 1998, 102, 5766.

(53) Keffer, D. Chem. Eng. J. 1999, 74, 33.

(54) Fan, X.; Dickey, E. C.; Eklund, P.; Williams, K.; Grigorian, L.;

Buczko, R.; Pantelides, S. T.; Pennycook, S. J. Phys. Rev. Lett., in press. 